\title{
Assessment of Hemodialysis Adequacy in patients with Chronic Kidney Disease in the Hemodialysis Unit at Sohag University Hospital
}

\author{
AUTHORS \\ Abdallah Hafez abdel-Naiem, Lotfy Hamed Abo Dahab, Eman Ahmed Sabet, \\ Emad Ahmed Mohammed
}

\section{ABSTRACT \\ INTRODUCTION}

evaluation of HD adequacy in patients with ESRD who were being maintained on regular HD in a trial to identify the prevalence and causes of inadequate HD among the patients and the impact of HD adequacy on other parameters.

DESIGN AND SETTING:PROSPECTIVE STUDY, AT SOHAG UNIVERSITY HOSPITAL IN EGYPT.

\section{METHODS}

All patients in this study were subjected to laboratory investigations including blood urea nitrogen (BUN) (before dialysis and after dialysis), serum albumin, and hemoglobin $(\mathrm{Hb})$ level.

- $\mathbf{U R R}=(1$ - [postdialysis $\mathrm{BUN} \div$ predialysis BUN] $)$

$\mathrm{Kt} / \mathrm{V}$ was calculated using the second-generation Daugirdas formula

- Single-pool Kt/V = $\mathbf{I n}(R-0.008 \times t)+(4-3.5 \times R) \times \mathrm{UF} / W$,

$R$ is the ratio of postdialysis to predialysis BUN; $t$ is the length of a dialysis session in hours; UF is the ultrafiltration volume in liters; and $W$ is the patient's postdialysis weight in kilograms.

\section{RESULTS}

The results shown that $69,64 \%$ of patients had adequate HD (KT\V >1,2), and only $30,36 \%$ of patients had indequate HD (KT\V>1,2).

\section{CONCLUSION}

A significant percentage $(69,64 \%)$ of patients in the HD unit of Sohag University hospital had adequate HD. HD adequacy was influenced by several factors such as duration and frequency of the dialysis session.

\section{INTRODUCTION}

End-stage renal disease (ESRD) causes irreversible, severe kidney failure for which patients require treatment with dialysis or kidney transplantation to survive [1].

It is a major outcome of chronic kidney disease (CKD), with an important effect on the quality of life (QOL) and health resource utilization [2]. Hemodialysis (HD) is one of the main modalities of renal replacement therapy [3]. HD patients still have higher mortality and hospitalization rates, as well as lower QOL, compared with the general population [4].

the adequacy of the dialysis dose has a profound effect on patient morbidity and mortality [5]. To ensure that patients are receiving the prescribed urea clearance, the clinician must regularly monitor and measure the dose delivered. Urea clearance should be measured at least every 8 wk. Examples of acceptable techniques for estimating delivered dose are formal single-pool urea kinetics, urea reduction ratio (URR), and $\mathrm{Kt} / \mathrm{V}$ natural logarithm formulae. [6].

URR stands for urea reduction ratio, meaning the reduction in urea as a result of dialysis. The URR is one measure of how effectively a dialysis treatment removed waste products 
from the body and is commonly expressed as a percentage.

Patients and methods

- Patients:

- Inclusion criteria:

- Patients on regular HD for 6 months (at least) by means of an arterio-venous fistula (AVF) in the hemodialysis unit at Sohag University Hospital

- $\quad$ Exclusion criteria:

○ Patients who are not on regular HD.

○ Patients using central venous catheters or arterio-venous grafts

- Patients with advanced comorbid conditions like advanced liver cirrhosis or heart failure or malignancy

-Method of the study: All patients in this study were subjected to full history taking, complete physical examination, and laboratory investigations including blood urea nitrogen (BUN) (before
$\mathrm{Kt} / \mathrm{V}$ is another way of measuring dialysis adequacy.

dialysis and after dialysis), serum albumin, and hemoglobin ( $\mathrm{Hb}$ ) level Urea reduction ratio (URR) was calculated using the following formula

- URR $=(1-$ [postdialysis BUN $\div$ predialysis BUN])

$\mathrm{Kt} / \mathrm{V}$ was calculated using the secondgeneration Daugirdas formula

- Single-pool Kt/V $=-\operatorname{In}(R-0.008 \times t)$ $+(4-3.5 \times R) \times \mathrm{UF} / W$,

Where In represents the natural logarithm; $R$ is the ratio of postdialysis to predialysis BUN; $t$ is the length of a dialysis session in hours; UF is the ultrafiltration volume in liters; and $W$ is the patient's postdialysis weight in kilograms. Online medical calculators will be used.

HD prescription was revised to detect the following:

(1) Blood flow rate (BFR).

(2) Ultrafiltration volume (UF volume)

(3) Effective surface area of the dialyzer.

(4)Venous pressure.

\section{The Result of the study}

our study included 112 patient classified into 2 groups

Group I: $\mathrm{Kt} / \mathrm{V}$ of at least 1.2 (adequate dialysis dose)

Group II: Kt/V less than 1.2 (inadequate dialysis dose) (34)

Comparison between $\mathrm{Kt} / \mathrm{V}$ values as regard blood flow rate

Astrong association between higher clearance rate and $\operatorname{BFR}((>250 \mathrm{ml} / \mathrm{min})$

$$
(\mathrm{p}=.0001)
$$

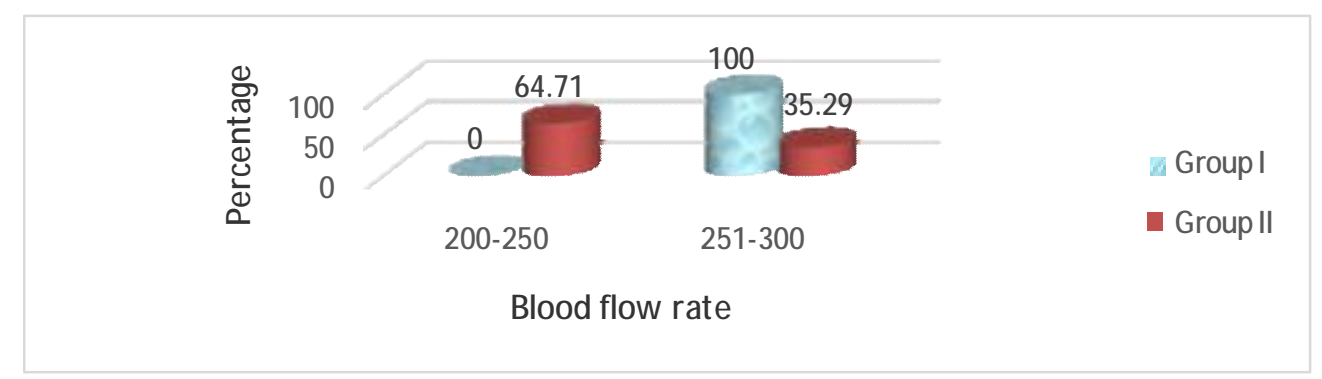

Comparison between $\mathrm{Kt} / \mathrm{V}$ values as regard UF volume: 
Decrease in clearance rate was noted in patients with UF volume greater than 31 as only $12.82 \%$ of them showed $\mathrm{Kt} / \mathrm{V}$ values of at least 1.2. Diff erences in the clearance rates were statistically insignificant $(P=0.80)$

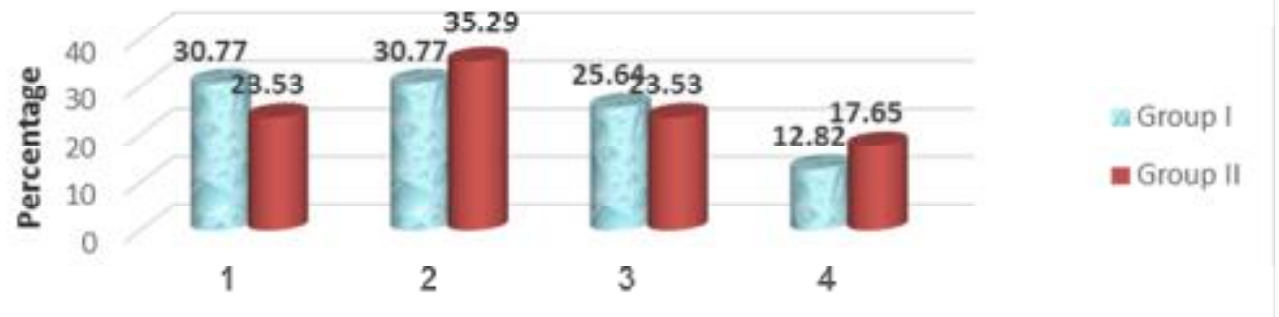

Comparison between $\mathrm{Kt} / \mathrm{V}$ values as regard duration of session:A strong association between higher clearance rates and increased dialysis duration of each session

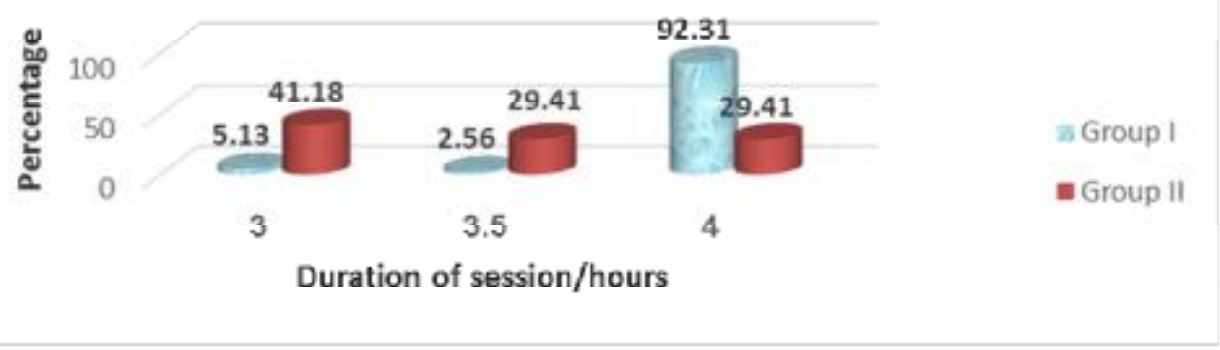

\section{Comparison between $\mathrm{Kt} / \mathrm{V}$ values as regard dialysis sessions per week:}

A strong association between higher clearance rates and frequency of dialysis per week

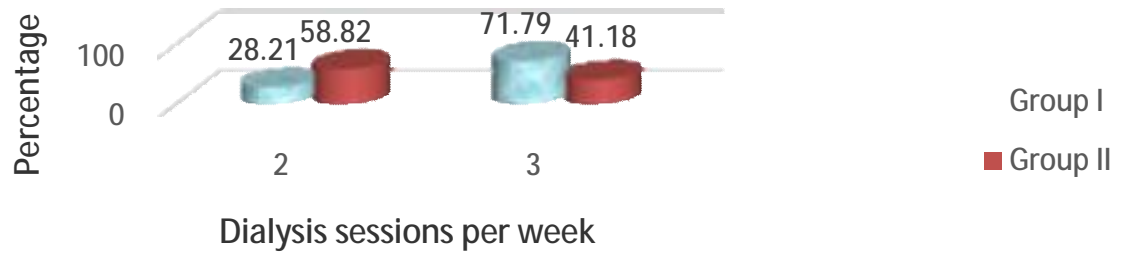

\section{Discussion}

It was noted that dialysis adequacy differs among the different dialysis centers.

It was reported to be high as in the USA, as, according to the 2007 annual report, over $90 \%$ of patients had a $\mathrm{Kt} / \mathrm{V}$ greater than 1.2 [5].

Other dialysis centers in other countries such as Brazil, Nigeria, Nepal, Pakistan, and Iran reported lower values of adequacy(only 35-
$45 \%$ of patients had a Kt/V greater than 1.2) [6a].

An Egyptian Study performed in Tanta in 2015 had Shown that $40 \%$ of patients had a $\mathrm{Kt} / \mathrm{V}$ greater than 1.2 [6b] .

As regards the relationship between $\mathrm{Kt} / \mathrm{V}$ and URR, the results of the present study revealed that all patients with spKt/V at least 1.2 had URR of at least $65 \%$ (statistically significant 
positive correlation betweenKt/V and URR; $P<0.0001)$. These results were in agreement with the study by Afshar et al. [7], who found a statistically strong correlation between URR and $\mathrm{eKt} / \mathrm{V}(P<0.001)$.

our results were in disagreement with the study by Oreo and Hamburger [8], who reported a poor correlation between URR and Kt/V in 942 patients when both values were measured simultaneously

As regards the BFR, analysis of the results of the present study revealed that increased BFRs were associated with increased rate of clearance. This is clear from the findings of $\mathrm{Kt} / \mathrm{V}$ values of at least $1.2(200-250 \mathrm{ml} / \mathrm{min}$, 0\%; 251-300 $\mathrm{ml} / \mathrm{min}, 100 \%$ ). Diff erence in clearance rates among the various groups of BFRs was statistically significant $(P=0.0001)$. These results were in agreement with the study by Kim et al. [9], Borzou et al. [10], Ward [11], and Port et al. [12].

our results were in disagreement with those of Ghali and Malik [13]; there was no significant effect of increasing BFR on HD adequacy. They attributed their results to the effect of other factors affecting dialysis adequacy, such as malnutrition, anemia, short time of dialysis session, premature cessation of sessions of HD, infection, inadequate blood flow from vascular access, hypotension episodes, technical reasons, and the design of the study and the sample size.

As regards the duration of HD sessions, analysis of the results of the present study revealed that clearance was strongly associated with increased duration of the dialysis process. Difference in clearance rates among the various duration periods was statistically significant $(P=0.0001)$. These results were in agreement with the study by Stewart et al. [14], who showed that time still had a profound effect on dialysis adequacy, indicating the importance of ensuring that patients remain on dialysis for the full time prescribed [5].

As regards dialysis frequency, analysis of the results of the present study revealed improvement in clearance rates with increased dialysis frequency per week.

\section{Conclusion}

A significant percentage $(69,64 \%)$ of patients in the HD unit of Sohag University hospital had adequate HD. HD adequacy was influenced by several factors such as duration and frequency of the dialysis session.

\section{References}

1. Krame r A, Ste 1 V, Zoccal i C, Hea f J, Ansel 1 D, Grönhagen-Riska C, et al. ERA-EDTA Registry An update on renal replacement therapy in Europe: ERA-EDTA Registry data from 1997 to 2006. Nephrol Dial Transplant 2009; 24:3557-66.

2.Vanholde $r$ R, Davenpor $t \quad A$, Hannedouch e T, Kooma n J, Kribbe n A, Lameire $\mathrm{N}$, et al., Dialysis Advisory Group of American Society of Nephrology Reimbursement of dialysis: a comparison of seven countries. J Am Soc Nephrol 2012; 23:129 1-8

3. Aghigh i M, Heidary Rouch i A, Zamyad i M, et al.: Dialysis in Iran. Iran J Kidney Dis 2008; 2:1 1-5.

4. Hall YN, Jolly SE, Abrass CK, et al.. Regional differences in dialysis care and mortality among American Indians and Alaska Natives. J Am Soc Nephrol 2011; 22:2287 -95.

5. Gotch FA, Sargent JA. A mechanistic analysis of the National

Cooperative Dialysis Study (NCDS). Kidney Int $1985 ; 28: 526-34$.

6. Plantinga LC, Fink NE, Jaar BG, et al.: Frequency of sit-down patient care rounds, attainment of clinical performance targets, hospitalization, 
SOHAG MEDICAL JOURNAL

Vol. 22 No.1 Jan 2018

and mortality in hemodialysis

patients. J Am Soc Nephrol 15:3144-
Assessment of Hemodialysis Adequacy in patients Abdallah Hafez abdel-Naiem

3153, 2004. 